\title{
Weighted Composition Operators between the Fractional Cauchy Spaces and the Bloch-Type Spaces
}

\author{
R. A. Hibschweiler \\ Department of Mathematics and Statistics, University of New Hampshire, Durham, NH 03824, USA \\ Correspondence should be addressed to R. A. Hibschweiler; rah2@unh.edu
}

Received 31 August 2017; Accepted 16 October 2017; Published 12 November 2017

Academic Editor: Sergei Grudsky

Copyright (c) 2017 R. A. Hibschweiler. This is an open access article distributed under the Creative Commons Attribution License, which permits unrestricted use, distribution, and reproduction in any medium, provided the original work is properly cited.

We characterize boundedness and compactness of weighted composition operators mapping the families of fractional Cauchy transforms into the Bloch-type spaces. Corollaries are obtained about composition operators and multiplication operators.

\section{Introduction}

Let $D$ denote the open unit disc in the complex plane and let $H(D)$ denote the space of functions analytic in $D$. Let $M$ denote the Banach space of complex-valued Borel measures on $T=\{\zeta:|\zeta|=1\}$, endowed with the total variation norm. For $\alpha>0$, the space $F_{\alpha}$ of fractional Cauchy transforms is the collection of functions of the form

$$
f(z)=\int_{T} \frac{1}{(1-\bar{\zeta} z)^{\alpha}} d \mu(\zeta) \quad(z \in D),
$$

where $\mu \in M$. The principal branch of the logarithm is used here. The space $F_{\alpha}$ is a Banach space, with norm given by

$$
\|f\|_{F_{\alpha}}=\inf \|\mu\| \text {, }
$$

where $\mu$ varies over all measures in $M$ for which (1) holds. The families $F_{\alpha}$ have been studied extensively $[1,2]$.

Let $\beta>0$. The Bloch-type space $B^{\beta}$ is the Banach space of functions analytic in $D$ such that $\sup _{z \in D}\left(1-|z|^{2}\right)^{\beta}\left|f^{\prime}(z)\right|<$ $\infty$, with norm

$$
\|f\|_{B^{\beta}}=|f(0)|+\sup _{z \in D}\left(1-|z|^{2}\right)^{\beta}\left|f^{\prime}(z)\right| .
$$

The integral representation (1) implies that $F_{\alpha} \subset B^{\alpha+1}$ and there is a constant depending only on $\alpha$ such that

$$
\|f\|_{B^{\alpha+1}} \leq C\|f\|_{F_{\alpha}}
$$

for $f \in F_{\alpha}$.
It is known that any univalent $f \in H(D)$ belongs to $F_{\alpha}$ for any $\alpha>2$. MacGregor [3] constructed a univalent function $f$ such that $f \notin F_{2}$. Let $g$ denote the normalized function $g=$ $(f-f(0)) / f^{\prime}(0)$. Then $g \notin F_{2}$. Since $g \in S$, the classical family of schlicht functions, the Distortion Theorem [4] yields $g \in$ $B^{3}$.

Let $\Phi$ be an analytic self-map of $D$ and let $u \in H(D)$. The weighted composition operator $W_{u, \Phi}$ is defined for $f \in H(D)$ by

$$
\left(W_{u, \Phi} f\right)(z)=u(z) f(\Phi(z)) \quad(z \in D) .
$$

If $u=1$, then the operator $W_{u, \Phi}$ reduces to the composition operator $C_{\Phi}$ defined by $C_{\Phi}(f)=f \circ \Phi$. If $\Phi$ is the identity function, then $W_{u, \Phi}$ is the multiplication operator $M_{u}$ defined by $M_{u}(f)=u f$.

This paper characterizes $\Phi$ and $u$ for which $W_{u, \Phi}: F_{\alpha} \rightarrow$ $B^{\beta}$ is bounded or compact. Corollaries are obtained for the operators $C_{\Phi}$ and $M_{u}$.

\section{Boundedness}

We follow the convention that $C$ denotes a positive constant, which may vary from one appearance to the next.

Theorem 1. Fix $\alpha>0$ and $\beta>0$. Let $u \in H(D)$ and let $\Phi$ be an analytic self-map of $D$. If

$$
\sup _{w \in D}\left|u^{\prime}(w)\right| \frac{\left(1-|w|^{2}\right)^{\beta}}{\left(1-|\Phi(w)|^{2}\right)^{\alpha}}<\infty,
$$




$$
\sup _{w \in D}|u(w)| \frac{\left(1-|w|^{2}\right)^{\beta}}{\left(1-|\Phi(w)|^{2}\right)^{\alpha+1}}\left|\Phi^{\prime}(w)\right|<\infty
$$

then $W_{u, \Phi}: F_{\alpha} \rightarrow B^{\beta}$ is bounded.

Proof. Assume the hypotheses. By a theorem due to Ohno et al., $W_{u, \Phi}: B^{\alpha+1} \rightarrow B^{\beta}$ is bounded [5]. Thus there is a constant $C$ such that $\left\|W_{u, \Phi}(f)\right\|_{B^{\beta}} \leq C\|f\|_{B^{\alpha+1}}$ for all $f \in B^{\alpha+1}$. Relation (4) now yields $\left\|W_{u, \Phi}(f)\right\|_{B^{\beta}} \leq C\|f\|_{F_{\alpha}}$ for all $f \in F_{\alpha}$.

The focus of this paper is to prove the converse of Theorem 1. Several lemmas are needed. Proofs of Lemmas 2 and 3 appear in [2].

Lemma 2. Fix $\alpha>0$ and let $w \in D$. Let $f_{w}(z)=1 /(1-\bar{w} z)^{\alpha}$ for $z \in D$. Then $f_{w} \in F_{\alpha}$ and $\left\|f_{w}\right\|_{F_{\alpha}}=1$.

Lemma 3. Fix $\alpha>0$ and let $f \in H(D)$. If $f^{\prime} \in F_{\alpha+1}$, then $f \in F_{\alpha}$ and there is a positive constant $C$ independent of $f$ such that

$$
\|f\|_{F_{\alpha}} \leq C\left\|f^{\prime}\right\|_{F_{\alpha+1}}+|f(0)|
$$

The first statement in Lemma 4 is due to MacGregor [3]. The norm inequality is due to Hibschweiler and Nordgren [6].

Lemma 4. Let $\alpha, \beta>0$. If $f \in F_{\alpha}$ and $g \in F_{\beta}$, then $f g \in F_{\alpha+\beta}$ and

$$
\|f g\|_{F_{\alpha+\beta}} \leq\|f\|_{F_{\alpha}}\|g\|_{F_{\beta}} .
$$

Lemma 5 will be used to develop test functions needed for the proof of the converse.

Lemma 5. Fix $\alpha>0$. Let $w \in D$ and define

$$
k_{w}(z)=\frac{1-|w|^{2}}{(1-\bar{w} z)^{\alpha+1}} \quad(z \in D) .
$$

Then $k_{w} \in F_{\alpha}$ and there is a constant $C$ independent of $w$ such that $\left\|k_{w}\right\|_{F_{\alpha}} \leq C$ for all $w \in D$.

Proof. First assume $\alpha=1$ and fix generic $w \in D$. A calculation shows that $k_{w}$ is in the Hardy space $H^{1}$ and $\left\|k_{w}\right\|_{H^{1}} \leq$ 1. Since the inclusion $H^{1} \subset F_{1}$ is bounded, this case is complete.

Fix $\alpha>1$. Then

$$
k_{w}(z)=\frac{1-|w|^{2}}{(1-\bar{w} z)^{2}} \frac{1}{(1-\bar{w} z)^{\alpha-1}} \quad(z \in D) .
$$

By the case for $\alpha=1$ and Lemma 2, $k_{w}$ is the product of a function in $F_{1}$ and a function in $F_{\alpha-1}$. By Lemma $4, k_{w} \in F_{\alpha}$ and there is a constant $C$ independent of $w$ such that $\left\|k_{w}\right\|_{F_{\alpha}} \leq$ $C$ for all $w$.

Finally fix $\alpha, 0<\alpha<1$. By the previous case,

$$
k_{w}^{\prime}(z)=\frac{(\alpha+1) \bar{w}\left(1-|w|^{2}\right)}{(1-\bar{w} z)^{\alpha+2}} \in F_{\alpha+1}
$$

and $\left\|k_{w}^{\prime}\right\|_{F_{\alpha+1}} \leq C$ for all $w$. By Lemma $3, k_{w} \in F_{\alpha}$ and $\left\|k_{w}\right\|_{F_{\alpha}} \leq$ $C$. The proof is complete.

We now prove the converse of Theorem 1. The test functions used in the proof first appeared in [5], in the context of the spaces $B^{\alpha+1}$.

Theorem 6. Fix $\alpha>0$ and $\beta>0$. Let $u \in H(D)$ and let $\Phi$ be an analytic self-map of $D$. Assume that $W_{u, \Phi}: F_{\alpha} \rightarrow B^{\beta}$ is bounded. Then

$$
\begin{aligned}
& C_{1}=\sup _{w \in D} \frac{\left|u^{\prime}(w)\right|\left(1-|w|^{2}\right)^{\beta}}{\left(1-|\Phi(w)|^{2}\right)^{\alpha}}<\infty, \\
& C_{2}=\sup _{w \in D} \frac{|u(w)|\left|\Phi^{\prime}(w)\right|\left(1-|w|^{2}\right)^{\beta}}{\left(1-|\Phi(w)|^{2}\right)^{\alpha+1}}<\infty .
\end{aligned}
$$

Proof. Fix $\alpha, \beta, u$, and $\Phi$ as described. By assumption there is a constant $C$ independent of $f$ such that

$$
\left\|W_{u, \Phi}(f)\right\|_{B^{\beta}} \leq C\|f\|_{F_{\alpha}}
$$

for all $f \in F_{\alpha}$.

The argument will first establish that $C_{1}<\infty$. Let $w \in D$ and define

$$
g_{w}(z)=\frac{\alpha+1}{(1-\overline{\Phi(w)} z)^{\alpha}}-\frac{\alpha\left(1-|\Phi(w)|^{2}\right)}{(1-\overline{\Phi(w)} z)^{\alpha+1}}
$$

$(z \in D)$

By Lemmas 2 and 5 , there is a constant $C$ such that $\left\|g_{w}\right\|_{F_{\alpha}} \leq C$ for all $w \in D$. Therefore

$$
\begin{aligned}
& \sup _{z \in D}\left(1-|z|^{2}\right)^{\beta} \\
& \cdot\left|u^{\prime}(z) g_{w}(\Phi(z))+u(z) g_{w}^{\prime}(\Phi(z)) \Phi^{\prime}(z)\right| \\
& \quad \leq\left\|W_{u, \Phi}\left(g_{w}\right)\right\|_{B^{\beta}} \leq C
\end{aligned}
$$

for all $w \in D$. Since

$$
\begin{aligned}
& g_{w}(\Phi(w))=\frac{1}{\left(1-|\Phi(w)|^{2}\right)^{\alpha}}, \\
& g_{w}^{\prime}(\Phi(w))=0,
\end{aligned}
$$

it follows that

$$
C_{1}=\sup _{w \in D} \frac{\left|u^{\prime}(w)\right|\left(1-|w|^{2}\right)^{\beta}}{\left(1-|\Phi(w)|^{2}\right)^{\alpha}}<\infty .
$$

In particular, (17) yields $u \in B^{\beta}$.

To obtain the second condition in the theorem, let $w \in D$ and define

$$
h_{w}(z)=\frac{1-|\Phi(w)|^{2}}{(1-\overline{\Phi(w)} z)^{\alpha+1}} \quad(z \in D) .
$$


By Lemma 5, there is a constant $C$ independent of $w$ such that $\left\|h_{w}\right\|_{F_{\alpha}} \leq C$. Relation (13) yields

$$
\begin{aligned}
& \sup _{z \in D}\left(1-|z|^{2}\right)^{\beta} \\
& \cdot\left|u^{\prime}(z) h_{w}(\Phi(z))+u(z) h_{w}^{\prime}(\Phi(z)) \Phi^{\prime}(z)\right| \\
& \quad \leq\left\|W_{u, \Phi}\left(h_{w}\right)\right\|_{B^{\beta}} \leq C
\end{aligned}
$$

for all $w \in D$. Since

$$
\begin{aligned}
& h_{w}(\Phi(w))=\frac{1}{\left(1-|\Phi(w)|^{2}\right)^{\alpha}}, \\
& h_{w}^{\prime}(\Phi(w))=\frac{(\alpha+1) \overline{\Phi(w)}}{\left(1-|\Phi(w)|^{2}\right)^{\alpha+1}}
\end{aligned}
$$

it follows that

$$
\begin{aligned}
& \left(1-|w|^{2}\right)^{\beta} \\
& \cdot\left|\frac{u^{\prime}(w)}{\left(1-|\Phi(w)|^{2}\right)^{\alpha}}+\frac{(\alpha+1) u(w) \Phi^{\prime}(w) \overline{\Phi(w)}}{\left(1-|\Phi(w)|^{2}\right)^{\alpha+1}}\right| \\
& \quad \leq C
\end{aligned}
$$

for all $w \in D$.

First consider $w \in D$ with $1 / 2<|\Phi(w)|$. By the triangle inequality, relation (21) yields

$$
\begin{aligned}
& \frac{\alpha+1}{2} \frac{\left(1-|w|^{2}\right)^{\beta}|u(w)|\left|\Phi^{\prime}(w)\right|}{\left(1-|\Phi(w)|^{2}\right)^{\alpha+1}} \\
& \leq \frac{(\alpha+1)\left(1-|w|^{2}\right)^{\beta}|u(w)|\left|\Phi^{\prime}(w)\right||\Phi(w)|}{\left(1-|\Phi(w)|^{2}\right)^{\alpha+1}} \\
& \leq C+\frac{\left(1-|w|^{2}\right)^{\beta}\left|u^{\prime}(w)\right|}{\left(1-|\Phi(w)|^{2}\right)^{\alpha}}
\end{aligned}
$$

for such $w$. By relation (17), it follows that

$$
\sup _{1 / 2<|\Phi(w)|} \frac{\left(1-|w|^{2}\right)^{\beta}|u(w)|\left|\Phi^{\prime}(w)\right|}{\left(1-|\Phi(w)|^{2}\right)^{\alpha+1}}<\infty .
$$

Finally consider $w \in D$ with $|\Phi(w)| \leq 1 / 2$. Let $f(z)=z$ in relation (13). Thus $u \Phi \in B^{\beta}$ and

$$
\begin{aligned}
& \left(1-|w|^{2}\right)^{\beta}\left|u(w) \Phi^{\prime}(w)+u^{\prime}(w) \Phi(w)\right| \\
& \quad \leq\left\|W_{u, \Phi}(z)\right\|_{B^{\beta}} \leq C
\end{aligned}
$$

for all $w \in D$. Therefore

$$
\begin{aligned}
& \left(1-|w|^{2}\right)^{\beta}\left|u(w) \Phi^{\prime}(w)\right| \\
& \quad \leq C+\left(1-|w|^{2}\right)^{\beta}\left|u^{\prime}(w) \Phi(w)\right| \leq C+\|u\|_{B^{\beta}}
\end{aligned}
$$

for all $w \in D$. Therefore

$$
\sup _{|\Phi(w)| \leq 1 / 2} \frac{\left(1-|w|^{2}\right)^{\beta}|u(w)|\left|\Phi^{\prime}(w)\right|}{\left(1-|\Phi(w)|^{2}\right)^{\alpha+1}}<\infty .
$$

Relations (23) and (26) yield

$$
C_{2}=\sup _{w \in D} \frac{|u(w)|\left|\Phi^{\prime}(w)\right|\left(1-|w|^{2}\right)^{\beta}}{\left(1-|\Phi(w)|^{2}\right)^{\alpha+1}}<\infty
$$

and the proof is complete.

Let $\gamma, \beta>0$. Ohno et al. [5] characterized $u$ and $\Phi$ for which $W_{u, \Phi}: B^{\gamma} \rightarrow B^{\beta}$ is bounded. Theorems 1 and 6 and their result yield the following corollary.

Corollary 7. Fix $\alpha, \beta>0$. Let $u \in H(D)$ and let $\Phi$ be an analytic self-map.

$$
\begin{gathered}
W_{u, \Phi}: F_{\alpha} \longrightarrow B^{\beta} \text { is bounded } \Longleftrightarrow \\
W_{u, \Phi}: B^{\alpha+1} \longrightarrow B^{\beta} \text { is bounded. }
\end{gathered}
$$

Xiao [7] characterized the self-maps $\Phi$ for which $C_{\Phi}$ : $B^{\gamma} \rightarrow B^{\beta}$ is bounded for $\gamma, \beta>0$.

Corollary 8. Fix $\alpha, \beta$, and $\Phi$ as above.

$$
\begin{aligned}
& C_{\Phi}: F_{\alpha} \longrightarrow B^{\beta} \text { is bounded } \Longleftrightarrow \\
& C_{\Phi}: B^{\alpha+1} \longrightarrow B^{\beta} \text { is bounded } \Longleftrightarrow \\
& \sup _{w \in D} \frac{\left(1-|w|^{2}\right)^{\beta}\left|\Phi^{\prime}(w)\right|}{\left(1-|\Phi(w)|^{2}\right)^{\alpha+1}}<\infty .
\end{aligned}
$$

Proof. The equivalence of the first two conditions follows from Corollary 7. The equivalence of the second and third conditions is due to Xiao.

Let $\gamma, \beta>0$ and let $u \in H(D)$. The function $u$ is a multiplier of $B^{\gamma}$ into $B^{\beta}$ if $M_{u}(f)=u f \in B^{\beta}$ for every $f \in B^{\gamma}$. By the Closed Graph Theorem, it follows that $M_{u}: B^{\gamma} \rightarrow B^{\beta}$ is bounded. The collection of all such multipliers is denoted $M\left(B^{\gamma}, B^{\beta}\right)$. In [5], Ohno et al. characterized $u \in M\left(B^{\gamma}, B^{\beta}\right)$.

Let $M\left(F_{\alpha}, B^{\beta}\right)$ denote the set of analytic functions $u$ for which $M_{u}: F_{\alpha} \rightarrow B^{\beta}$ is bounded. Corollary 9 follows from Corollary 7 and the characterization in [5] for the case $\gamma=$ $\alpha+1>1$.

Corollary 9. Fix $\alpha, \beta>0$ and let $u \in H(D)$.

$$
M\left(F_{\alpha}, B^{\beta}\right)= \begin{cases}B^{\beta-\alpha}, & \text { if } \beta>\alpha+1, \\ H^{\infty}, & \text { if } \beta=\alpha+1, \\ \{0\}, & \text { if } \beta<\alpha+1 .\end{cases}
$$




\section{Compactness}

A characterization is given for functions $u$, $\Phi$ for which $W_{u, \Phi}$ : $F_{\alpha} \rightarrow B^{\beta}$ is compact.

Lemma 10. Fix $\alpha>0$ and let $w \in D$. Define $L_{w} b y$

$$
L_{w}(z)=\frac{\left(1-|w|^{2}\right)^{2}}{(1-\bar{w} z)^{\alpha+2}} \quad(z \in D) .
$$

Then $L_{w} \in F_{\alpha}$ and there is a constant $C$ such that $\left\|L_{w}\right\|_{F_{\alpha}} \leq C$ for all $w \in D$.

Proof. First fix $\alpha=2$ and let $w \in D$. A particular case of Lemma 5 provides a constant $C$ independent of $w \in D$ such that

$$
\left\|\frac{1-|w|^{2}}{(1-\bar{w} z)^{2}}\right\|_{F_{1}} \leq C
$$

for all $w \in D$. Since

$$
L_{w}(z)=\left(\frac{1-|w|^{2}}{(1-\bar{w} z)^{2}}\right)^{2}
$$

Lemma 4 now implies that $L_{w} \in F_{2}$ and $\left\|L_{w}\right\|_{F_{2}} \leq C$ for all $w$.

When $\alpha>2$,

$$
L_{w}(z)=\frac{\left(1-|w|^{2}\right)^{2}}{(1-\bar{w} z)^{4}} \frac{1}{(1-\bar{w} z)^{\alpha-2}} .
$$

By the previous case and Lemma 2, $L_{w}$ is the product of a function in $F_{2}$ and a function in $F_{\alpha-2}$. By Lemma $4, L_{w} \in F_{\alpha}$ and $\left\|L_{w}\right\|_{F_{\alpha}} \leq C$ for all $w \in D$.

Fix $\alpha, 1 \leq \alpha<2$. By the previous cases $L_{w}^{\prime} \in F_{\alpha+1}$ and $\left\|L_{w}^{\prime}\right\|_{F_{\alpha+1}} \leq C$ for all $w \in D$. Lemma 3 shows that $L_{w} \in F_{\alpha}$ and $\left\|L_{w}\right\|_{F_{\alpha}}^{\alpha+1} \leq C$. A similar argument applies when $0<\alpha<1$. The proof is complete.

Lemma 11 is the standard sequential criterion for compactness.

Lemma 11. Fix $\alpha, \beta>0$. The operator $W_{u, \Phi}: F_{\alpha} \rightarrow B^{\beta}$ is compact if and only if $\left\|W_{u, \Phi}\left(f_{n}\right)\right\|_{B^{\beta}} \rightarrow 0$ as $n \rightarrow \infty$ for any sequence $\left(f_{n}\right)$ in $F_{\alpha}$ with $\left\|f_{n}\right\|_{F_{\alpha}} \leq C$ and $f_{n} \rightarrow 0$ uniformly on compact subsets of $D$ as $n \rightarrow \infty$.

Theorem 12. Fix $\alpha, \beta>0$. Assume that $W_{u, \Phi}: F_{\alpha} \rightarrow B^{\beta}$ is bounded. The operator $W_{u, \Phi}: F_{\alpha} \rightarrow B^{\beta}$ is compact if and only if

$$
\begin{gathered}
\lim _{|\Phi(w)| \rightarrow 1} \frac{\left|u^{\prime}(w)\right|\left(1-|w|^{2}\right)^{\beta}}{\left(1-|\Phi(w)|^{2}\right)^{\alpha}}=0, \\
\lim _{|\Phi(w)| \rightarrow 1} \frac{|u(w)|\left|\Phi^{\prime}(w)\right|\left(1-|w|^{2}\right)^{\beta}}{\left(1-|\Phi(w)|^{2}\right)^{\alpha+1}}=0 .
\end{gathered}
$$

Proof. Fix $\alpha, \beta>0$ and assume that $W_{u, \Phi}: F_{\alpha} \rightarrow B^{\beta}$ is bounded.

First assume the limit conditions (35) and (36). Corollary 7 implies that $W_{u, \Phi}: B^{\alpha+1} \rightarrow B^{\beta}$ is bounded and it now follows as in [5] that $W_{u, \Phi}: B^{\alpha+1} \rightarrow B^{\beta}$ is compact. Suppose that $\left(f_{n}\right)$ is a sequence in $F_{\alpha}$ such that $\left\|f_{n}\right\|_{F_{\alpha}} \leq C$ for all $n$ and $f_{n} \rightarrow 0$ uniformly on compact subsets. By relation (4), $\left\|f_{n}\right\|_{B^{\alpha+1}} \leq C$ and thus $\left\|W_{u, \Phi}\left(f_{n}\right)\right\|_{B^{\beta}} \rightarrow 0$ as $n \rightarrow \infty$. By Lemma $11, W_{u, \Phi}: F_{\alpha} \rightarrow B^{\beta}$ is compact.

Now assume that $W_{u, \Phi}: F_{\alpha} \rightarrow B^{\beta}$ is compact. We may assume that $\|\Phi\|_{\infty}=1$. Let $\left(w_{n}\right)$ be any sequence in $D$ with $\left|\Phi\left(w_{n}\right)\right| \rightarrow 1$ as $n \rightarrow \infty$. For $n=1,2, \ldots$ define

$$
h_{n}(z)=\frac{1-\left|\Phi\left(w_{n}\right)\right|^{2}}{\left(1-\overline{\Phi\left(w_{n}\right) z}\right)^{\alpha+1}} \quad(z \in D) \text {. }
$$

By Lemma $5,\left\|h_{n}\right\|_{F_{\alpha}} \leq C$ for all $n$. Also $h_{n} \rightarrow 0$ uniformly on compact subsets of $D$ as $n \rightarrow \infty$. Thus $\left\|W_{u, \Phi}\left(h_{n}\right)\right\|_{B^{\beta}} \rightarrow 0$ as $n \rightarrow \infty$ and

$$
\begin{aligned}
& \sup _{w \in D}\left(1-|w|^{2}\right)^{\beta} \\
& \cdot\left|u^{\prime}(w) h_{n}(\Phi(w))+u(w) h_{n}^{\prime}(\Phi(w)) \Phi^{\prime}(w)\right| \\
& \quad \longrightarrow 0
\end{aligned}
$$

as $n \rightarrow \infty$. Calculations yield

$$
\begin{aligned}
& \left(1-\left|w_{n}\right|^{2}\right)^{\beta} \mid \frac{u^{\prime}\left(w_{n}\right)}{\left(1-\left|\Phi\left(w_{n}\right)\right|^{2}\right)^{\alpha}} \\
& +\frac{(\alpha+1) u\left(w_{n}\right) \Phi^{\prime}\left(w_{n}\right) \overline{\Phi\left(w_{n}\right)}}{\left(1-\left|\Phi\left(w_{n}\right)\right|^{2}\right)^{\alpha+1}} \mid \rightarrow 0
\end{aligned}
$$

as $n \rightarrow \infty$.

The argument will first establish that

$$
\frac{\left(1-\left|w_{n}\right|^{2}\right)^{\beta}\left|u^{\prime}\left(w_{n}\right)\right|}{\left(1-\left|\Phi\left(w_{n}\right)\right|^{2}\right)^{\alpha}} \longrightarrow 0
$$

as $n \rightarrow \infty$. As in [5], define the test functions

$$
\begin{aligned}
f_{n}(z)= & \frac{(\alpha+2)\left(1-\left|\Phi\left(w_{n}\right)\right|^{2}\right)}{\left(1-\overline{\Phi\left(w_{n}\right) z}\right)^{\alpha+1}} \\
& -\frac{(\alpha+1)\left(1-\left|\Phi\left(w_{n}\right)\right|^{2}\right)^{2}}{\left(1-\overline{\Phi\left(w_{n}\right)} z\right)^{\alpha+2}},
\end{aligned}
$$

where $z \in D$ and $n=1,2, \ldots$. Then $f_{n} \rightarrow 0$ uniformly on compact subsets as $n \rightarrow \infty$. By Lemmas 10 and 5, there 
is a constant $C$ with $\left\|f_{n}\right\|_{F_{\alpha}} \leq C$ for all $n$. It now follows that

$$
\begin{aligned}
& \sup _{z \in D}\left(1-|z|^{2}\right)^{\beta} \\
& \cdot\left|u^{\prime}(z) f_{n}(\Phi(z))+u(z) f_{n}^{\prime}(\Phi(z)) \Phi^{\prime}(z)\right| \\
& \quad \leq\left\|W_{u, \Phi}\left(f_{n}\right)\right\|_{B^{\beta}} \longrightarrow 0 \text { as } n \longrightarrow \infty .
\end{aligned}
$$

In particular,

$$
\begin{aligned}
& \left(1-\left|w_{n}\right|^{2}\right)^{\beta} \mid u^{\prime}\left(w_{n}\right) f_{n}\left(\Phi\left(w_{n}\right)\right) \\
& \quad+u\left(w_{n}\right) f_{n}^{\prime}\left(\Phi\left(w_{n}\right)\right) \Phi^{\prime}\left(w_{n}\right) \mid \longrightarrow 0
\end{aligned}
$$

as $n \rightarrow \infty$.

$$
\begin{aligned}
& \text { Since } f_{n}\left(\Phi\left(w_{n}\right)\right)=\frac{1}{\left(1-\left|\Phi\left(w_{n}\right)\right|^{2}\right)^{\alpha}} \\
& \text { since } f_{n}^{\prime}\left(\Phi\left(w_{n}\right)\right)=0
\end{aligned}
$$

relation (40) is established. Since $\left(w_{n}\right)$ is a generic sequence with $\left|\Phi\left(w_{n}\right)\right| \rightarrow 1$ as $n \rightarrow \infty$, relation (35) holds. yield

To complete the proof note that relations (39) and (40)

$$
\left(1-\left|w_{n}\right|^{2}\right)^{\beta} \frac{\left|u\left(w_{n}\right)\right|\left|\Phi^{\prime}\left(w_{n}\right)\right|\left|\Phi\left(w_{n}\right)\right|}{\left(1-\left|\Phi\left(w_{n}\right)\right|^{2}\right)^{\alpha+1}} \longrightarrow 0
$$

as $n \rightarrow \infty$. Since $\left|\Phi\left(w_{n}\right)\right| \rightarrow 0$,

$$
\left(1-\left|w_{n}\right|^{2}\right)^{\beta} \frac{\left|u\left(w_{n}\right)\right|\left|\Phi^{\prime}\left(w_{n}\right)\right|}{\left(1-\left|\Phi\left(w_{n}\right)\right|^{2}\right)^{\alpha+1}} \longrightarrow 0
$$

as $n \rightarrow \infty$. Condition (36) follows and the proof is complete.

Corollary 13. Fix $\alpha, \beta>0$ and assume that $W_{u, \Phi}: F_{\alpha} \rightarrow B^{\beta}$ is bounded.

$$
\begin{gathered}
W_{u, \Phi}: F_{\alpha} \rightarrow B^{\beta} \text { is compact } \Longleftrightarrow \\
W_{u, \Phi}: B^{\alpha+1} \longrightarrow B^{\beta} \text { is compact. }
\end{gathered}
$$

Proof. The hypothesis and Corollary 7 yield that $W_{u, \Phi}$ : $B^{\alpha+1} \rightarrow B^{\beta}$ is bounded.

Assume that $W_{u, \Phi}: B^{\alpha+1} \rightarrow B^{\beta}$ is compact. Since the inclusion $F_{\alpha} \subset B^{\alpha+1}$ is bounded, it follows that $W_{u, \Phi}: F_{\alpha} \rightarrow$ $B^{\beta}$ is compact.

Assume that $W_{u, \Phi}: F_{\alpha} \rightarrow B^{\beta}$ is compact. By Theorem 12, conditions (35) and (36) hold. These conditions are sufficient to imply that the bounded operator $W_{u, \Phi}: B^{\alpha+1} \rightarrow B^{\beta}$ is compact [5].

Let $\gamma, \beta>0$ and assume that $C_{\Phi}: B^{\gamma} \rightarrow B^{\beta}$ is bounded. In [7], Xiao provided additional conditions on $\Phi$ necessary and sufficient for $C_{\Phi}: B^{\gamma} \rightarrow B^{\beta}$ to be compact.
Corollary 14. Fix $\alpha, \beta>0$ and assume that $C_{\Phi}: F_{\alpha} \rightarrow B^{\beta}$ is bounded. The following are equivalent:

(1) $C_{\Phi}: F_{\alpha} \rightarrow B^{\beta}$ is compact.

(2) $C_{\Phi}: B^{\alpha+1} \rightarrow B^{\beta}$ is compact.

(3) $\lim _{|\Phi(w)| \rightarrow 1}\left(\left(1-|w|^{2}\right)^{\beta}\left|\Phi^{\prime}(w)\right| /\left(1-|\Phi(w)|^{2}\right)^{\alpha+1}\right)=0$.

Proof. Corollary 13 yields the equivalence of the first and second conditions.

Since $C_{\Phi}: F_{\alpha} \rightarrow B^{\beta}$ is bounded, Corollary 8 yields that $C_{\Phi}: B^{\alpha+1} \rightarrow B^{\beta}$ is bounded. Under this hypothesis, Xiao [7] proved the equivalence of the second and third conditions.

Fix $\gamma, \beta>0$. In [5], Ohno et al. characterized $u$ for which the bounded operator $M_{u}: B^{\gamma} \rightarrow B^{\beta}$ is compact.

Let $u \in H(D)$ and let $\beta>0$. Recall that $u$ is in the little Bloch space $B_{0}^{\beta}$ if

$$
\lim _{|z| \rightarrow 1}\left(1-|z|^{2}\right)^{\beta}\left|u^{\prime}(z)\right|=0
$$

Corollary 13 and the characterization in [5] for $\gamma=\alpha+1>$ 1 yield the following result.

Corollary 15. Fix $\alpha, \beta>0$ and assume $M_{u}: F_{\alpha} \rightarrow B^{\beta}$ is bounded.

(1) Assume $\beta>\alpha+1 . M_{u}: F_{\alpha} \rightarrow B^{\beta}$ is compact $\Leftrightarrow u \epsilon$ $B_{0}^{\beta-\alpha}$.

(2) Assume $\beta \leq \alpha+1 . M_{u}: F_{\alpha} \rightarrow B^{\beta}$ is compact $\Leftrightarrow u \equiv 0$.

\section{Conflicts of Interest}

The author declares that there are no conflicts of interest regarding the publication of this paper.

\section{References}

[1] J. A. Cima, A. L. Matheson, and W. T. Ross, The Cauchy transform, Mathematical Surveys and Monographs, American Mathematical Society, Providence, RI, USA, 2006.

[2] R. A. Hibschweiler and T. H. MacGregor, Fractional Cauchy transforms, CRC Press, Boca Raton, Fla, USA, 2006.

[3] T. H. MacGregor, "Analytic and univalent functions with integral representations involving complex measures," Indiana University Mathematics Journal, vol. 36, no. 1, pp. 109-130, 1987.

[4] P. L. Duren, Univalent Functions, Springer-Verlag, New York, NY, USA, 1983.

[5] S. Ohno, K. Stroethoff, and R. Zhao, "Weighted composition operators between Bloch-type spaces," Rocky Mountain Journal of Mathematics, vol. 33, no. 1, pp. 191-215, 2003.

[6] R. Hibschweiler and E. Nordgren, "Cauchy transforms of measures and weighted shift operators on the disc algebra," Rocky Mountain Journal of Mathematics, vol. 26, no. 2, pp. 627654, 1996.

[7] J. Xiao, "Composition operators associated with Bloch-type spaces," Complex Variables, Theory and Application, vol. 46, no. 2, pp. 109-121, 2001. 


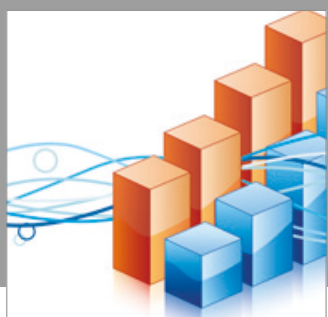

Advances in

Operations Research

vatersals

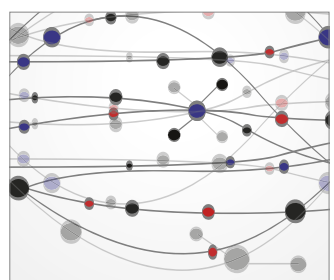

\section{The Scientific} World Journal
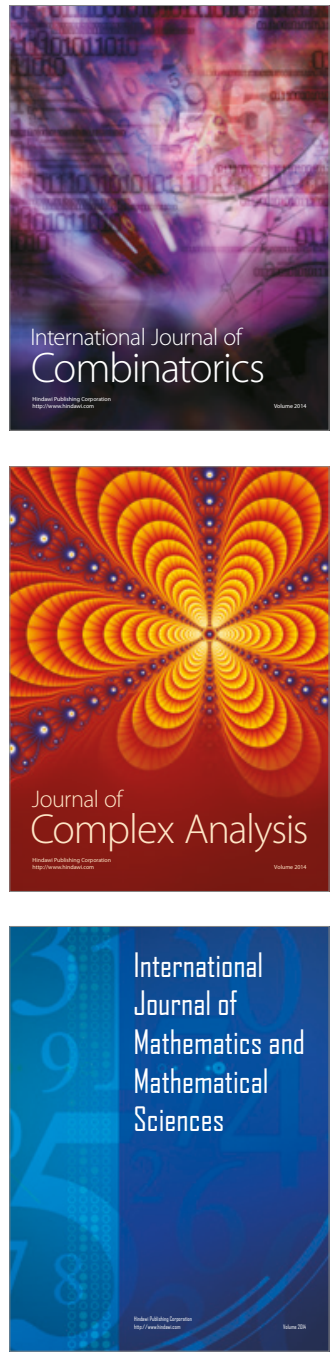
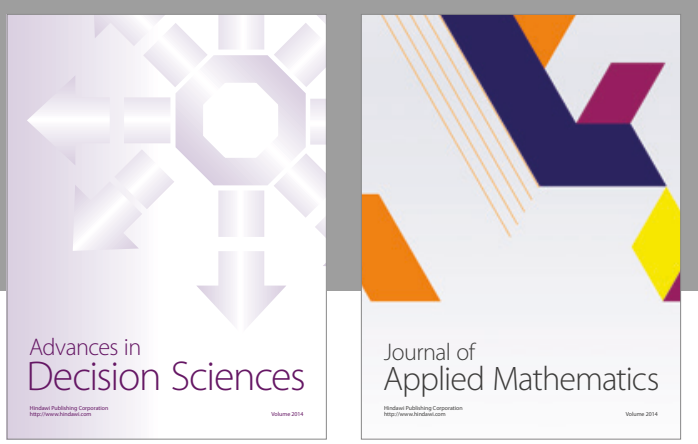

Algebra

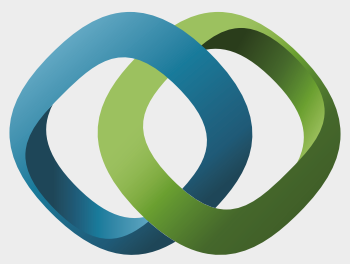

\section{Hindawi}

Submit your manuscripts at

https://www.hindawi.com
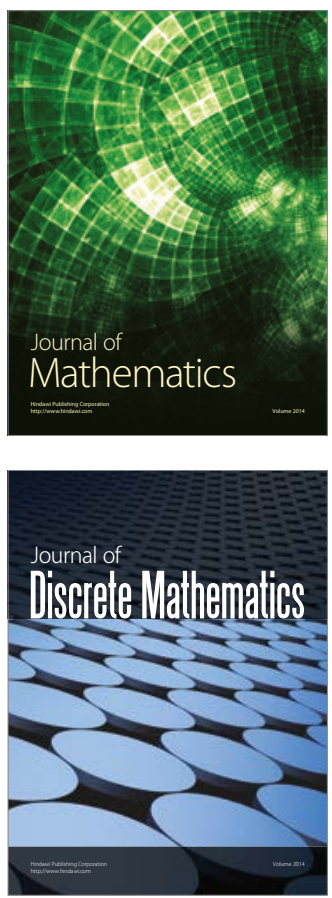

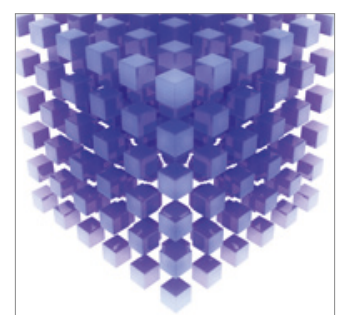

Mathematical Problems in Engineering
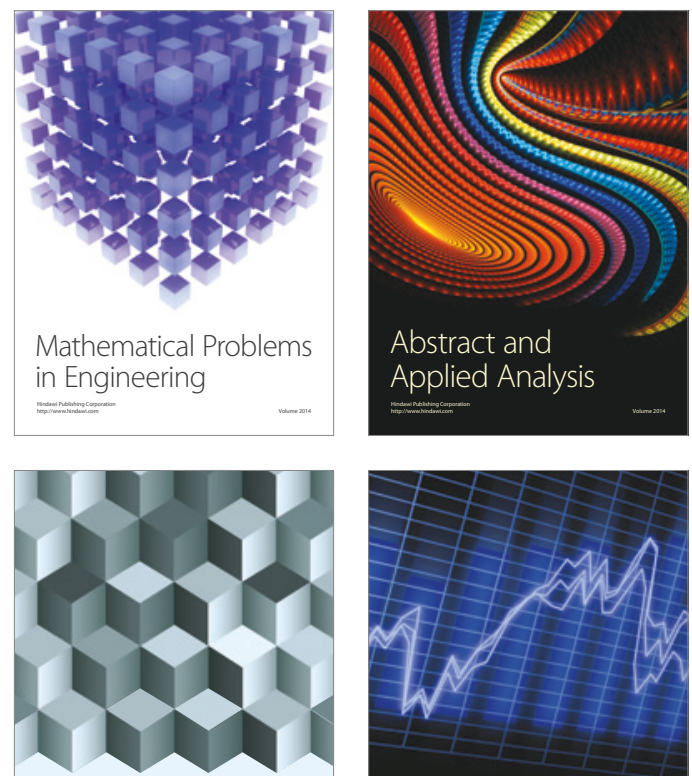

Journal of

Function Spaces

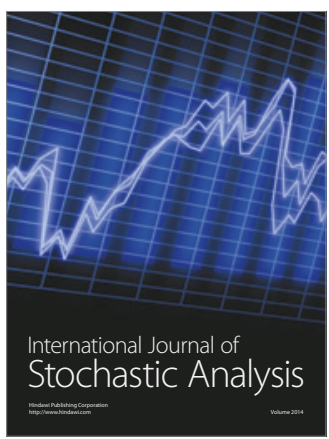

Probability and Statistics
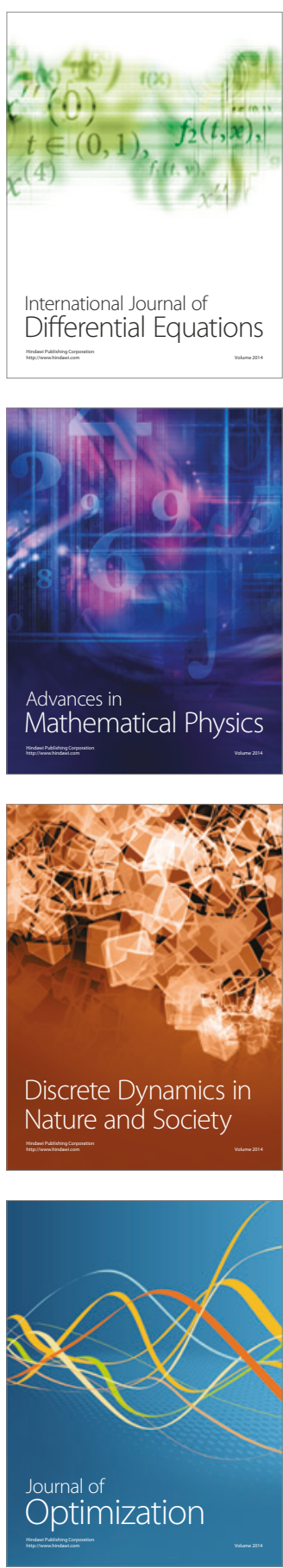